\title{
Free Since 1983
}

Like a celebratory burst of confetti, the colorful nuclei on this month's cover herald the arrival of the international edition of BioTechniques. For the first time, life scientists in Europe, Asia, the Middle East, and all of North America will be eligible for complimentary subscriptions to 12 full issues of BioTechniques each year. The growth of the journal's subscriber community to international proportions is a result of two factors. First, it is a response to the enormous and enduring popularity of the journal as a forum for the dissemination of techniques and technical tools applied across the gamut of life science research. Second, and equally important, it is testimony to the robust nature of the advertiser-supported model of peer-reviewed science publishing.

Based on two tenets - that technology drives discovery and that research data are interpretable only in the context of the techniques used to generate them - the central focus of BioTechniques articles has always been the technique. Methods are described in precise detail, accompanied by that amount of validating data necessary to clearly demonstrate the efficacy of the method. This format has been extremely effective in communicating techniques in such a way that readers can implement the methodology directly or adapt it to their particular experimental systems.

Long before the open access debate moved into the labs and lunchrooms of research institutions everywhere, BioTechniques was available free of charge. The journal was introduced in 1983 and the concept of a peerreviewed publication supported by advertising immediately caught on. Scientists appreciated the opportunity to access useful, high-quality methods free of charge, and advertisers saw the advantages of directly reaching interested, technically oriented readers. The value to readers over these many years is impressive to consider: one similarly ranked methods journal costs over $\$ 2000$ per year for a personal subscription. A BioTechniques reader pays nothing, and an author has the satisfaction of knowing that his/her article will reach more professional life scientists (over 85,000) than any other peer-reviewed journal.

Online access to content is, of course, critical, and the journal web site, www.BioTechniques.com, has been newly renovated to accompany the launch of the international print edition. At the site, full content is freely available to all subscribers and site registrants back to 2000 as HTML and PDF, and back to 1996 as PDF. Users will continue to see increased functionality as the site evolves.

The BioTechniques model can continue to thrive as it has for 22 years only with the participation of the BioTechniques community of authors, reviewers, and readers. We acknowledge our Authors for recognizing the added value and high visibility that accompany publication in BioTechniques. Because BioTechniques articles focus exclusively on the techniques rather than the elaboration of findings obtained using the methods described, readers may not be cognizant of the authors' long-term research goals and where the published technique fits into the "big picture." We hope to fill this gap with the introduction of a new monthly feature, About the Authors (p.152). Reviewers are an integral component of our community, and we thank them for the 1500 manuscript reviews we received last year; their comments are critical for maintaining and tightening our editorial standards. Because more than $75 \%$ of our reviewers return their comments on time (within 7 days), BioTechniques can offer authors one of the fastest turnaround times in science publishing, with an average time to first decision after review of just 23 days. Readers support the journal, when employing or adapting a technique published in BioTechniques, by including a citation to the article in their own articles. Although methods articles are cited less frequently than those published in other categories of journals (consider the impact factors of the journals ranked by ISI in the category "biochemical research methods"), citations benefit both the authors and the journal. Readers further support BioTechniques by mentioning the journal when making purchases based on articles or advertising seen in BioTechniques. Although we as editors maintain strict editorial independence, our advertisers are an integral part of the BioTechniques model and deserve support.

Finally, the Editors welcome feedback. Send your comments and recommendations for content to us at bioeditor@BioTechniques.com and join us in continuing to make BioTechniques the definitive journal for methods, tools, and life science resources for scientists around the world. 